\title{
Association of FKBP5 genotype with depressive symptoms in patients with coronary heart disease: a prospective study
}

\author{
Julia Brandt ${ }^{1}\left[\right.$ D Katharina Warnke ${ }^{1} \cdot$ Silke Jörgens ${ }^{1} \cdot$ Volker Arolt $^{1} \cdot$ Katja Beer $^{2} \cdot$ Katharina Domschke $^{3}$. \\ Wilhelm Haverkamp ${ }^{4}$. Stella L. Kuhlmann ${ }^{5,6}$. Jacqueline Müller-Nordhorn ${ }^{6,7}$. Nina Rieckmann ${ }^{6} \cdot$ Kathrin Schwarte $^{1}$. \\ Andreas Ströhle $^{2} \cdot$ Mira Tschorn $^{2,8} \cdot$ Johannes Waltenberger $^{9} \cdot$ Laura Grosse $^{1,10}$
}

Received: 18 March 2020 / Accepted: 10 August 2020 / Published online: 29 August 2020

(c) The Author(s) 2020

\begin{abstract}
Depression and coronary heart disease (CHD) are prevalent and often co-occurring disorders. Both have been associated with a dysregulated stress system. As a central element of the stress system, the FKBP5 gene has been shown to be associated with depression. In a prospective design, this study aims to investigate the association of $F K B P 5$ with depressive symptoms in CHD patients. $N=268$ hospitalized CHD patients were included. Depressive symptoms were measured using the Hospital Anxiety and Depression Scale (HADS-D) at four time points (baseline, and after 1 month, 6 months, and 12 months). The functional FKBP5 single-nucleotide polymorphism (SNP) rs1360780 was selected for genotyping. Linear regression models showed that a higher number of FKBP5 C alleles was associated with more depressive symptoms in CHD patients both at baseline $(p=0.015)$ and at 12-months follow-up $(p=0.025)$ after adjustment for confounders. Further analyses revealed that this effect was driven by an interaction of FKBP5 genotype with patients' prior CHD course. Specifically, only in patients with a prior myocardial infarction or coronary revascularization, more depressive symptoms were associated with a higher number of $\mathrm{C}$ alleles (baseline: $p=0.046$; 1-month: $p=0.026$; 6-months: $p=0.028$ ). Moreover, a higher number of $\mathrm{C}$ alleles was significantly related to a greater risk for dyslipidemia $(p=.016)$. Our results point to a relevance of $F K B P 5$ in the association of the two stress-related diseases depression and CHD.
\end{abstract}

Keywords Depressive symptoms $\cdot$ FKBP5 $\cdot$ HPA axis $\cdot$ CHD $\cdot$ Gene environment interaction $\cdot$ Stress-related disease

Julia Brandt

juliabrandt@mailbox.org

1 Department of Psychiatry and Psychotherapy, University Hospital Münster, Albert-Schweitzer-Campus 1, Geb. A9, 48149 Münster, Germany

2 Charité-Universitätsmedizin Berlin, Corporate member of the Freie Universität Berlin, Humboldt-Universität zu Berlin, and the Berlin Institute of Health, Department of Psychiatry and Psychotherapy, Berlin, Germany

3 Department of Psychiatry and Psychotherapy, Medical Center-University of Freiburg, Faculty of Medicine, University of Freiburg, Freiburg, Germany

4 Charité-Universitätsmedizin Berlin, Corporate member of the Freie Universität Berlin, Humboldt-Universität zu Berlin, and the Berlin Institute of Health, Department of Internal Medicine and Cardiology, Berlin, Germany
5 , Charité-Universitätsmedizin Berlin, Corporate member of the Freie Universität Berlin, Humboldt-Universität $\mathrm{zu}$ Berlin, and the Berlin Institute of Health, Division of Emergency and Acute Medicine (CVK, CCM), Berlin, Germany

6 , Charité-Universitätsmedizin Berlin, Corporate member of the Freie Universität Berlin, Humboldt-Universität zu Berlin, Berlin Institute of Health, Institute of Public Health, Berlin, Germany

7 Bavarian Food and Health Safety Authority, Oberschleißheim, Germany

8 Social and Preventive Medicine, University of Potsdam, Potsdam, Germany

9 Department of Cardiology, Central Hospital, Suhl, Germany

10 Intercultural Business Psychology, Hamm-Lippstadt University of Applied Sciences, Hamm, Germany 


\section{Introduction}

Depression and coronary heart disease (CHD) are both highly prevalent disorders and are associated with increased morbidity, mortality, and cause substantial economic costs (GBD 2013 DALYs and HALE Collaborators et al. 2015; Whiteford et al. 2013). Additionally, both medical conditions frequently co-occur (Rudisch and Nemeroff 2003). The association between CHD and depression is thought to be bidirectional (Whooley and Wong 2013). The wide range of approaches explaining this association includes a dysregulation of the hypothalamic-pituitary-adrenal (HPA) axis, which occurs in both conditions (Holsboer 2000; Jokinen and Nordstrom 2009). The HPA axis is the major stress hormone system and, in fact, exposure to stressors has repeatedly been linked with the development of both depression and cardiovascular diseases (Cohen et al. 2007; Kendler et al. 1999). There is evidence for a genetic overlap between depression and $\mathrm{CHD}$, as a result of which the related (pleiotropic) genes might lead, e.g. over the shared biological pathways of a HPA axis dysregulation, to both diseases (Amare et al. 2017; McCaffery et al. 2009).

In recent years, the FKBP5 gene has gained increased scientific interest regarding the genetic vulnerability to depression. The FKBP5 gene codes for the FK506 binding protein 51 (FKBP5), a co-chaperone of the heat shock protein 90 (hsp90) that operates as an inhibitor of the glucocorticoid receptor (GR). The GR is a central element of the HPA axis and directs the body's stress reaction by affecting gene transcription (Nicolaides et al. 2014). As part of an ultra-short negative feedback loop, the GR also induces the expression of FKBP5. This negative feedback restrains the activity of the HPA axis and ensures the homeostasis of the stress response (Denny et al. 2000). Within the FKBP5 gene there is one haplotype which is described to be functional and which comprises several single nucleotide polymorphisms (SNPs) in high linkage disequilibrium, amongst others SNP rs1360780. The minor (rarer) $\mathrm{T}$ allele of this SNP is the high-induction allele of the FKBP5 gene transcription and thus likely associated with a reduced cortisol binding affinity to the GR, also termed as GR resistance. A GR resistance and related prolonged glucocorticoid elevation have been shown to be associated with depression (Pariante and Lightman 2008). Accordingly, healthy $\mathrm{T}$ allele carriers showed a prolonged cortisol response following exposure to minor stressors (Ising et al. 2008) and less suppression of cortisol in pharmacological suppression tests (Binder et al. 2008; Touma et al. 2011).

An increasing number of studies have investigated the association of common variants in FKBP5 and stressrelated disorders like depression, posttraumatic stress disorder (PTSD), and suicidal events (Zannas et al. 2016). However, the results regarding a genetic main effect of $F K B P 5$ on psychiatric disorders remain inconsistent (Hernandez-Diaz et al. 2019; Rao et al. 2016; Zannas et al. 2016).

The etiology of depression is considered to be polygenetic and multifactorial (Sullivan et al. 2012). Moreover, as no specific locus for depression could be unequivocally identified yet (Major Depressive Disorder Working Group of the Psychiatric GWAS Consortium et al. 2013), geneenvironment $(\mathrm{G} \times \mathrm{E})$ interactions have moved into focus (Caspi et al. 2010). There is a growing body of evidence for a $F K B P 5 \times$ stressor interaction to confer risk for depression, PTSD (Wang et al. 2018), and other psychiatric phenotypes (Zannas et al. 2016). In the majority of reports, an interactive association of the FKBP5 high induction alleles $\times$ childhood trauma with the respective phenotype was observed. Only few studies explored a $F K B P 5 \times \mathrm{E}$ interaction regarding life stressors beyond childhood traumata and the results across the different studies are inconsistent (Zannas and Binder 2014).

In the present study, we investigated a sample with elevated risk for depression, in particular a sample of $N=268$ hospitalized CHD patients. We aimed to (1) investigate whether FKBP5 genotype (rs 1360780) was associated with depressive symptoms in CHD patients and (2) to explore a possible $\mathrm{G} \times \mathrm{E}$ effect of $F K B P 5$ and experienced stressors on depressive symptoms. We expected that $F K B P 5$ genotype would be associated with depressive symptoms specifically in those CHD patients who had experienced a prior myocardial infarction (MI) or coronary revascularization entailing a more stressful CHD course. Additionally, patients were followed up and longitudinally analyzed for depressive symptoms at three time points after study inclusion (1 months, 6 months, 12 months).

\section{Experimental procedures}

\section{Design and participants}

Participants were recruited while treated at one of two recruitment sites in Münster, Germany (Department of Cardiology, University Hospital Münster) and Berlin, Germany (Department of Cardiology, Campus Virchow Clinic, Charité) between December 2012 and July 2014. We included $N=298$ patients diagnosed with CHD and with sufficient proficiency in German (Münster site) or German or Turkish (Berlin site). Patients with a chart-documented dementia disorder, severe cognitive impairments, or with a terminal disease were excluded. The study was approved by the respective ethics committees and was in accordance with the Declaration of Helsinki. After study 
procedures had been fully explained, all subjects provided written informed consent. Of the $N=298$ included subjects, $n=30$ were excluded post hoc after inclusion because of withdrawn consent, missing CHD diagnosis, cognitive impairments, missing questionnaire data, double inclusion, or missing genetic data. Accordingly, the final sample consisted of $N=268$ patients (Fig. 1).

The present prospective cohort study comprised several self-rate questionnaires and clinical data collection from the medical chart at baseline and a blood collection. Moreover, three follow-up assessments with questionnaires were conducted after 1, 6, and 12 months (Fig. 1).

\section{Clinical instruments}

\section{Assessment of depressive symptoms}

To assess depressive symptoms the self-rated, seven-item depression subscale of the Hospital Anxiety and Depression Scale (HADS-D, German adaption) (Zigmond and Snaith 1983) was used. The HADS-D was included in the baseline assessment as well as in all follow-up questionnaires. At all time points, patients were also asked whether they received a current antidepressive treatment (antidepressant medication or psychotherapy).

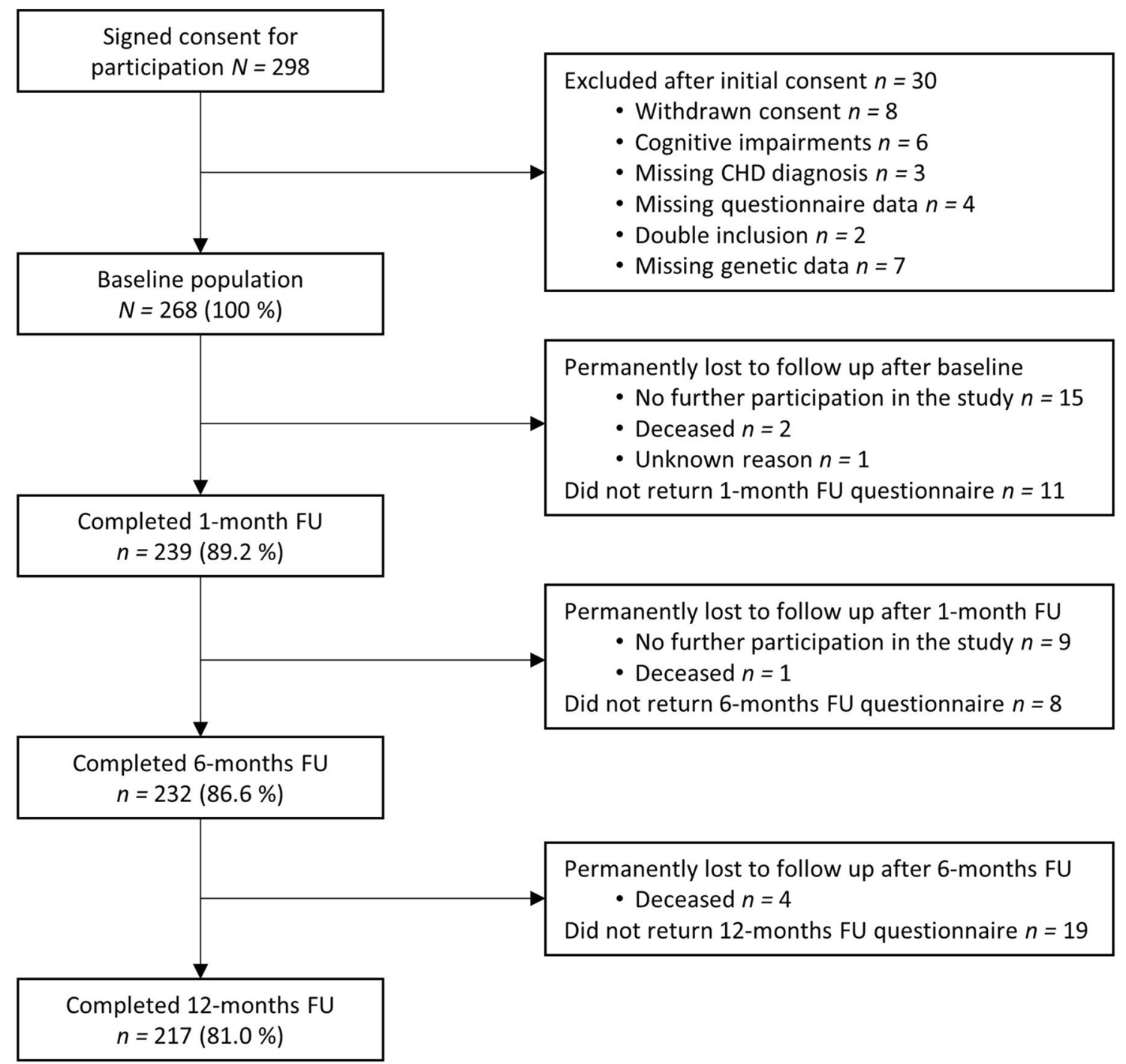

Fig. 1 Flow chart of patient selection. $C H D$ coronary heart disease, $F U$ follow-up. The figure shows the selection and exclusion of study participants, response rates, and drop-outs. Participants who perma- nently dropped out and those who further participated in the study after not having returned one or two questionnaires are listed separately 


\section{Coronary heart disease status and related risk factors}

To specify the patient's CHD course, it was assessed whether the patient had a prior MI or coronary revascularization. Therefore, the following information was recorded from the medical chart: MI, percutaneous coronary intervention, and coronary artery bypass grafting in his/her lifetime before study inclusion. Cardiac disease severity at baseline was assessed by left ventricular ejection fraction (LVEF) which was measured by electrocardiography, magnetic resonance imaging, or cardiac catheterisation or it was abstracted from the medical chart. Moreover, we distinguished CHD patients with and without an acute coronary syndrome (ACS) at study baseline (index hospitalization). The following CHD risk factors were assessed: diabetes, body mass index (BMI), and dyslipidemia according to the medical chart as well as current smoking (determined by both patient's self-report on the questionnaire and an additional patient interview led by the study nurse).

\section{Comorbidity}

The cumulative burden of physical comorbidity was assessed with the Charlson Comorbidity Index (CCI). The CCI is a measure for classifying comorbidity in longitudinal studies and predicting mortality outcomes from comorbid disease (Charlson et al. 1987). Since in our study, we were interested in somatic comorbidity other than cardiac diasease and diabetes, an important CHD risk factor (Greenland et al. 2003), we removed MI, congestive heart failure, and diabetes (which were all included in the original CCI version) from the CCI score. The CCI was used as continuous variable with higher scores indicating higher comorbidity.

\section{DNA extraction and SNP genotyping}

We collected $20 \mathrm{ml}$ of venous blood from each patient and stored it at $-20{ }^{\circ} \mathrm{C}$ until further use. The DNA of peripheral leukocytes was extracted using the FlexiGene DNA Kit (Qiagen, Hilden, Germany) in accordance with the manufacturer's protocol.

Genotyping of subjects for FKBP5 rs1360780 was performed using TaqMan SNP Genotyping Technology (Applied Biosystems by Life Technologies, Darmstadt, Germany) on an ABI prism 7000 Sequence Detection System. The $15 \mu \mathrm{l}$ final reaction mix consisted of $7.5 \mu \mathrm{l}$ TaqMan Universal PCR Master Mix, $0.375 \mu$ l Genotyping Assay (40×) containing 1 pair of target-specific primers and 1 pair of fluorescent probes, $4.125 \mu \mathrm{l}$ water and a total of $60 \mathrm{ng}$ DNA. All the assays were predesigned and validated (Applied Biosystems). DNA was amplified by polymerase chain reaction $\left(2 \mathrm{~min}\right.$ at $50^{\circ} \mathrm{C}, 10 \mathrm{~min}$ at $95^{\circ} \mathrm{C}, 15 \mathrm{~s}$ at $95^{\circ} \mathrm{C}$ for 40 cycles, $1 \mathrm{~min}$ at $60^{\circ} \mathrm{C}$ ) in 96 well plates. For allelic discrimination the Sequence Detection Software Version 1.5.1 (Applied Biosystems) was used. For quality control, duplicate genotyping was performed in $\sim 5 \%$ of the sample. No differences between duplicated analyses were observed. There was no deviation from Hardy-Weinberg equilibrium for FKBP5 rs1360780 genotype distribution $\left(\chi^{2}=0.92, p=0.34\right)$.

\section{Statistical analyses}

We used an additive model of linear regression (Binder et al. 2008), where the three genotypes were coded as 0,1 , or 2 according to the number of $\mathrm{C}$ alleles ( $\mathrm{TT}=0, \mathrm{CT}=1$, and $\mathrm{CC}=2$ ). Associations between genotypic distribution of the FKBP5 rs 1360780 and sample characteristics were analyzed using binary logistic regression for categorical data (e.g. gender, CHD course) and linear regression for continuous data (e.g. age, CCI).

Linear regression was used to analyze both a genetic main effect and an interaction effect of FKBP5 and the prior CHD course on self-rated depressive symptoms (HADS-D). Cronbach's alpha was calculated to assess the internal consistency of the HADS-D scale at the different timepoints. Based on theoretical considerations, we tested the following as potential covariates in our regression analyses using forward selection: age, gender, smoking, BMI, diabetes, dyslipidemia, physical comorbidity (CCI), LVEF, and current antidepressive treatment. In all analyses, differences were regarded as statistically significant at $p \leq 0.05$. Multiple imputation was performed to deal with missing data. Details have been described previously (Kuhlmann et al. 2017). Statistical analyses were performed using IBM SPSS version 24 for Windows (SPSS Inc., Chicago, Illinois).

\section{Results}

\section{Sample characteristics}

Patients' mean age at baseline was $63.5( \pm 10.2)$ years and $80.6 \%$ of the patients were of male sex $(n=216$; Table 1$)$. The most prevalent FKBP5 rs 1360780 genotype was CC $(n=141,52.6 \%)$, followed by the heterozygous genotype CT $(n=111,41.4 \%)$ and TT homozygosity $(n=16,6.0 \%)$. The minor allele frequency was 0.27 , comparable to HapMap data (the HapMap minor allele frequency for rs1360780: 0.27 in European samples).

With regard to CHD characteristics, $64.2 \%(n=172)$ of the patients had a prior MI or coronary revascularization in their lifetime before study inclusion (Table 1). At baseline, an ACS was diagnosed in 44.4\% $(n=119)$ of the patients. Patients' mean LVEF was $48.39 \%$ ( \pm 14.12$)$, indicating an overall mild dysfunction of the cardiac pump capacity. Regarding CHD risk factors, 26.9\% $(n=72)$ patients had 
Table 1 Sample and medical characteristics by FKBP5 rs1360780 genotype

\begin{tabular}{|c|c|c|c|c|c|}
\hline & \multirow{2}{*}{$\begin{array}{l}\text { Total sample } \\
N=268(100 \%)\end{array}$} & \multicolumn{3}{|c|}{ FKBP5 rs1360780 genotype } & \multirow[t]{2}{*}{$p^{1}$} \\
\hline & & $\begin{array}{l}\mathrm{CC} \\
n=141(52.6 \%)\end{array}$ & $\begin{array}{l}\text { CT } \\
n=111(41.4 \%)\end{array}$ & $\begin{array}{l}\mathrm{TT} \\
n=16(6.0 \%)\end{array}$ & \\
\hline \multicolumn{6}{|l|}{ Demographics } \\
\hline Age $M \pm \mathrm{SD}$ & $63.49 \pm 10.21$ & $63.79 \pm 10.33$ & $63.40 \pm 9.94$ & $61.50 \pm 11.31$ & 0.464 \\
\hline Male $n(\%)$ & $217(81.0)$ & $117(83.0)$ & $88(79.3)$ & $12(75.0)$ & 0.333 \\
\hline Charlson Comorbidity Index $(\mathrm{CCI})^{2} M \pm \mathrm{SD}$ & $0.81 \pm 1.23$ & $0.84 \pm 1.20$ & $0.82 \pm 1.32$ & $0.38 \pm 0.89$ & 0.318 \\
\hline \multicolumn{6}{|l|}{ Coronary heart disease (CHD) characteristics } \\
\hline Prior MI/coronary revascularization $n(\%)$ & $172(64.2)$ & $93(66.0)$ & $68(61.3)$ & $11(68.8)$ & 0.709 \\
\hline Acute coronary syndrome at baseline $n(\%)$ & $119(44.4)$ & $60(42.6)$ & $53(47.7)$ & $6(37.5)$ & 0.761 \\
\hline Left ventricular ejection fraction (\%) $M \pm S D$ & $48.39 \pm 14.12$ & $48.25 \pm 14.02$ & $48.81 \pm 14.47$ & $46.74 \pm 14.39$ & 0.964 \\
\hline \multicolumn{6}{|l|}{ CHD risk factors } \\
\hline Current smoking $n(\%)$ & $70(26.1)$ & $39(27.7)$ & $28(25.2)$ & $3(18.8)$ & 0.443 \\
\hline Diabetes $n(\%)$ & $72(26.9)$ & $37(26.2)$ & $29(26.1)$ & $6(37.5)$ & 0.558 \\
\hline Body mass index $\left(\mathrm{kg} / \mathrm{m}^{2}\right) M \pm \mathrm{SD}$ & $28.36 \pm 5.02$ & $28.02 \pm 4.81$ & $28.39 \pm 4.89$ & $31.19 \pm 6.85$ & 0.059 \\
\hline Dyslipidemia $n(\%)$ & $191(71.3)$ & $108(76.6)$ & $75(67.6)$ & $8(50.0)$ & 0.016 \\
\hline \multicolumn{6}{|l|}{ Antidepressive treatment } \\
\hline Baseline $n(\%)$ & $13(4.9)$ & $4(2.8)$ & $7(6.3)$ & $2(12.5)$ & 0.063 \\
\hline 1-month $n(\%)$ & $12(4.5)$ & $5(3.5)$ & $6(5.4)$ & $1(6.3)$ & 0.439 \\
\hline 6-months $n(\%)$ & $13(4.9)$ & $4(2.8)$ & $8(7.2)$ & $1(6.3)$ & 0.157 \\
\hline 12-months $n(\%)$ & $16(6.0)$ & $8(5.7)$ & $8(7.2)$ & - & 0.819 \\
\hline
\end{tabular}

$P$-values $<0.05$ were considered statistically significant and are shown in bold

$M$ mean, $S D$ standard deviation, $M I$ myocardial infarction

${ }^{1}$ We used an additive model with the three genotypes coding as 0 (TT), 1 (CT), or 2 (CC) according to the number of $\mathrm{C}$ alleles

${ }^{2}$ We used a modified CCI (excluded were cardiac diagnoses and diabetes)

The variable 'prior MI/coronary revascularization' includes MI, percutaneous coronary intervention, and coronary artery bypass grafting before study inclusion. For analyzing categorical data, logistic regression and for analyzing continuous variables, linear regression was used. FKBP5 rs1360780 genotype was significantly associated with dyslipidemia

diabetes and $71.3 \%(n=191)$ dyslipidemia. Patients' mean BMI $(28.36 \pm 5.02)$ indicated a tendency towards overweight and $n=70(26.1 \%)$ were current smokers (Table 1$)$. Analyses revealed that a higher number of $\mathrm{C}$ alleles of FKBP5 rs1360780 was significantly associated with dyslipidemia (odds ratio $[\mathrm{OR}]=1.69,95 \%$ confidence interval $[\mathrm{CI}]=1.10-2.61, p=0.016)$. No association of $F K B P 5$ rs1360780 with other sample characteristics, e.g. age, gender, or CHD course, was observed (Table 1). With regard to depression, patients' initial mean HADS-D score was 5.10 $( \pm 4.14$; Table 2$)$. The internal consistency of the questionnaire is satisfying for all timepoints (Cronbach's alpha $\mathrm{T} 0=0.86, \mathrm{~T} 1=0.87, \mathrm{~T} 2=0.87$, and $\mathrm{T} 3=0.89$ )

\section{FKBP5 rs1360780 genotype and depressive symptoms}

Regression analyses revealed that current depressive symptoms were significantly related to FKBP5 rs1360780 genotype, controlling for confounders (Table 2). In particular, controlling for LVEF, BMI, and antidepressive treatment, more depressive symptoms at study baseline $(p=0.015)$ and at 12-months follow-up ( $p=0.025)$ were significantly associated with a higher number of $\mathrm{C}$ alleles.

\section{FKBP5 rs1360780 genotype, depressive symptoms, and prior CHD course}

Considering the robust evidence regarding $F K B P 5 \times$ stressor interaction on the risk for depression (Zannas and Binder 2014), we performed a linear regression model to test for possible interaction of the $F K B P 5$ genotype and the prior CHD course on depressive symptoms. The analyses revealed that the above reported main effect of FKBP5 genotype on depressive symptoms (Table 2) was in fact driven by the interaction of FKBP5 genotype $\times$ prior CHD course (Table 3). In particular, in patients who had at least one myocardial infarction or coronary revascularization before and independent of the baseline hospitalization, more depressive symptoms were associated with a higher number of $\mathrm{C}$ alleles (Fig. 2). Figure 2 shows the effect of the number of $\mathrm{C}$ alleles on baseline depressive symptoms that occurred only 


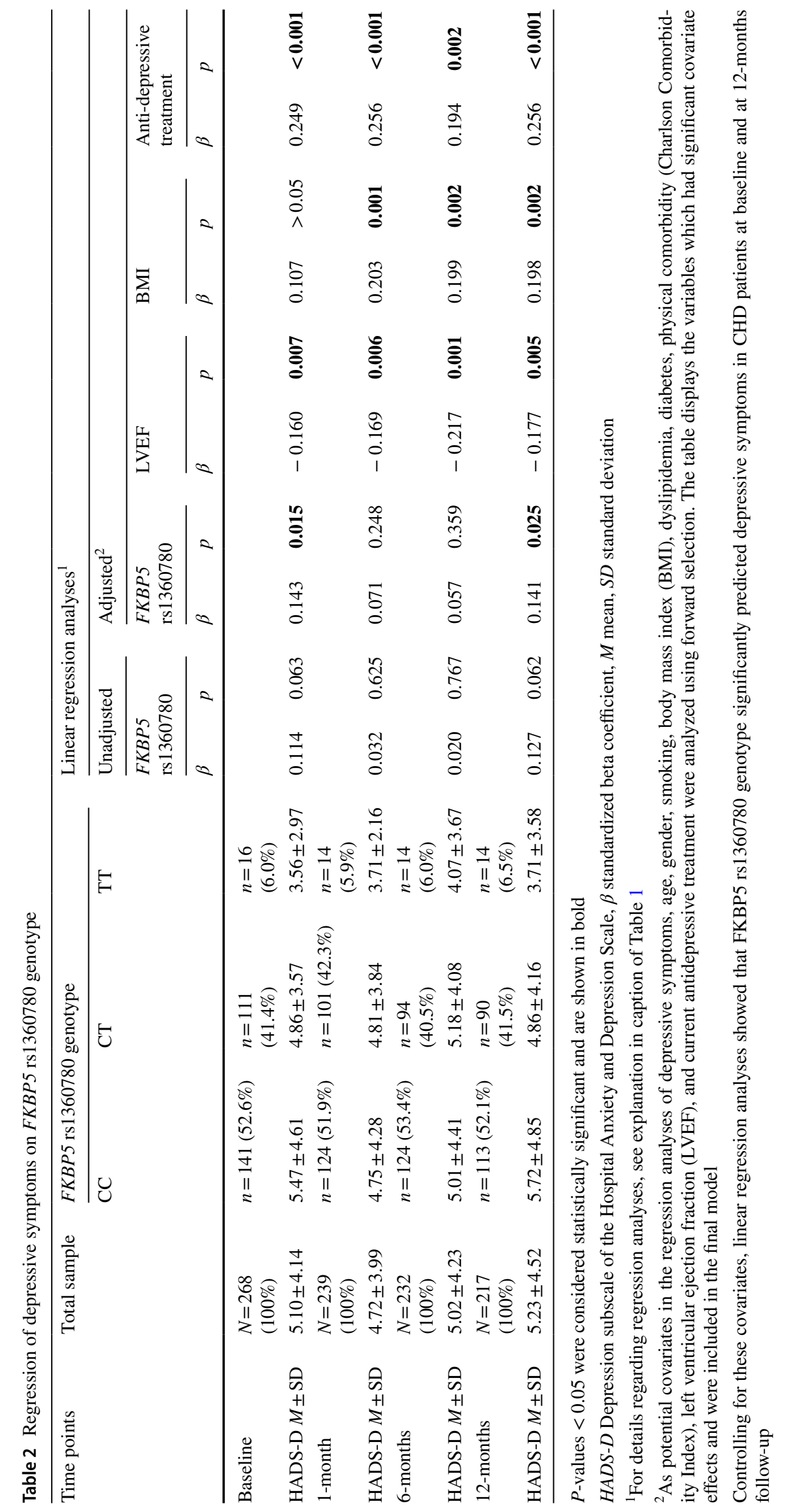


Table 3 Regression of depressive symptoms on the interaction between FKBP5 rs1360780 genotype and prior coronary heart disease (CHD) course

\begin{tabular}{|c|c|c|c|c|c|c|c|c|}
\hline \multirow[t]{3}{*}{ Depressive symptoms } & \multicolumn{6}{|c|}{ Included predictors } & \multirow{2}{*}{\multicolumn{2}{|c|}{ Total model }} \\
\hline & \multicolumn{2}{|c|}{ Genotype $^{1}$} & \multicolumn{2}{|c|}{ Prior CHD course ${ }^{2}$} & \multicolumn{2}{|c|}{$\begin{array}{l}\text { Genotype } \times \text { Prior CHD } \\
\text { course }\end{array}$} & & \\
\hline & $\beta$ & $p$ & $\beta$ & $p$ & $\beta$ & $p$ & $\operatorname{Var}^{3}$ & $p$ \\
\hline \multicolumn{9}{|l|}{ (A) Unadjusted models } \\
\hline Baseline & -0.093 & 0.364 & -0.202 & 0.199 & 0.439 & 0.015 & 0.047 & 0.001 \\
\hline 1-month follow-up & -0.178 & 0.113 & -0.212 & 0.219 & 0.450 & 0.021 & 0.037 & 0.008 \\
\hline 6-months follow-up & -0.180 & 0.108 & -0.232 & 0.180 & 0.425 & $\mathbf{0 . 0 3 3}$ & 0.020 & 0.057 \\
\hline 12-months follow-up & -0.013 & 0.911 & -0.196 & 0.269 & 0.296 & 0.142 & 0.014 & 0.108 \\
\hline \multirow[t]{3}{*}{ Depressive symptoms } & \multicolumn{6}{|c|}{ Included predictors } & \multirow{2}{*}{\multicolumn{2}{|c|}{ Total model }} \\
\hline & \multicolumn{2}{|l|}{ Genotype } & \multicolumn{2}{|c|}{ Prior CHD course } & \multicolumn{2}{|c|}{$\begin{array}{l}\text { Genotype } \times \text { Prior } \mathrm{CHD} \\
\text { course }\end{array}$} & & \\
\hline & $\beta$ & $p$ & $\beta$ & $p$ & $\beta$ & $p$ & Var & $p$ \\
\hline \multicolumn{9}{|l|}{ (B) Adjusted ${ }^{4}$ models } \\
\hline Baseline & -0.025 & 0.802 & -0.161 & 0.300 & 0.353 & 0.046 & 0.115 & $<0.001$ \\
\hline 1-month follow-up & -0.124 & 0.246 & -0.222 & 0.180 & 0.413 & 0.026 & 0.145 & $<0.001$ \\
\hline 6-months follow-up & -0.137 & 0.200 & -0.262 & 0.114 & 0.414 & 0.028 & 0.133 & $<0.001$ \\
\hline 12-months follow-up & -0.011 & 0.916 & -0.261 & 0.115 & 0.323 & 0.083 & 0.164 & $<0.001$ \\
\hline
\end{tabular}

$P$-values $<0.05$ were considered statistically significant and are shown in bold

$\beta$ standardized beta coefficient

${ }^{1}$ For details regarding regression analyses, see explanation in caption of Table 1

${ }^{2}$ Prior CHD course: $0=$ no prior myocardial infarction (MI)/coronary revascularization (percutaneous coronary intervention or coronary artery bypass grafting), $1=\geq 1$ prior $\mathrm{MI} /$ coronary revascularization

${ }^{3}$ Variance explained by total model

${ }^{4}$ For details regarding potential covariates, see explanation in caption of Table 2

The variables left ventricular ejection fraction, body mass index, and antidepressive treatment had significant covariate effects in several analyses and were included as covariates in the respective final model

The tables show a significant effect of FKBP5 rs1360780 genotype x Prior CHD course on depressive symptoms at baseline, 1 month follow-up, and 6 months follow-up

Fig. 2 Interaction of $F K B P 5$ rs1360780 genotype and prior coronary heart disease (CHD) course on baseline depressive symptoms. $M I$ myocardial infarction. Results indicate that a higher number of $\mathrm{C}$ alleles was associated with more depressive symptoms specifically in those CHD patients who have had a prior myocardial infarction or coronary revascularization (percutaneous coronary intervention or coronary artery bypass grafting). Unadjusted: $p=0.014, \beta=0.442$; Adjusted: $p=0.044, \beta=0.356$. The error bars reflect the standard error of the mean (SEM). The numbers in columns indicate the count of patients

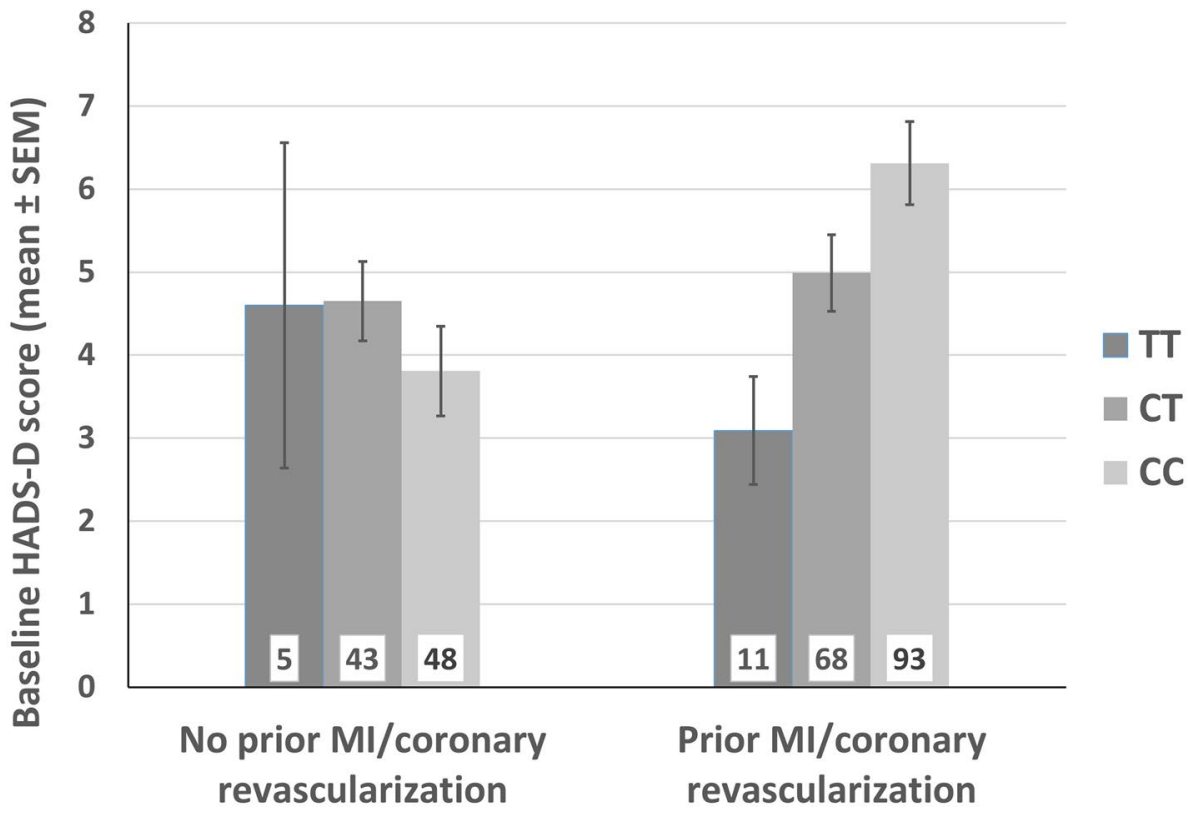


in those CHD patients with a prior MI or coronary revascularization. In contrast, the number of $\mathrm{C}$ alleles conferred no risk for depressive symptoms in CHD patients who had not experienced a CHD event before. This association was significant regarding the HADS-D scores at baseline and at 1 - and 6-months follow-up $(\beta=0.439, p=0.015 ; \beta=0.450$, $p=0.021$; and $\beta=0.425, p=0.033)$. After adjustment for confounders, the effect for baseline and 1- and 6-months depressive symptoms remained significant $(p=0.046$; $p=0.026 ; p=0.028)$ and showed a trend towards significance regarding 12-months depressive symptoms ( $p=0.083$; Table 3).

\section{Discussion}

In this study, we prospectively investigated relations of the FKBP5 rs1360780 genotype with depressive symptoms in hospitalized CHD patients. The current study shows that a higher number of $\mathrm{C}$ alleles was associated with more depressive symptoms in CHD patients at study baseline and at 12-months follow-up after adjustment for confounders. Moreover, this study indicates that this effect is driven by an interaction of the FKBP5 genotype with patients' CHD course. Specifically, in patients with a prior MI or coronary revascularization, the number of $\mathrm{C}$ alleles was associated with depressive symptoms.

In the literature, there is some evidence for a main effect of $F K B P 5$ polymorphisms on depression and other psychiatric disorders (Zannas et al. 2016). A recent meta-analysis found an association of FKBP5 rs1360780 with suicidal behavior but not with depressive disorders, whereas two other FKBP5 SNPs were associated with depressive disorders (Hernandez-Diaz et al. 2019). Considering the central role of stressor exposure for the association of FKBP5 and psychiatric disorders (Wang et al. 2018; Zannas et al. 2016), our findings point in the direction that, as a chronic disease, CHD in itself might act as stressor. Regarding other stress-responsive genes of the HPA axis and their role in the bidirectional association between CHD and depression, the following explanation has recently been proposed: the organism might consider CHD (respectively depression) as chronic stress and might induce functional regulatory changes in the stress-responsive genes of the HPA axis. The subsequent HPA axis dysregulation might increase the risk for depressive symptoms (respectively CHD) (Amare et al. 2017). Another interpretation might be that FKBP5 is a pleiotropic gene increasing the risk for both depression and CHD. This is supported by our findings, that a higher number of $\mathrm{C}$ alleles was not only associated with more depressive symptoms but also with higher risk for dyslipidemia, an important CHD risk factor (Greenland et al. 2003). Recent findings of a genetic overlap between mood disorders and cardiovascular disease included various genes which regulate the HPA axis (Amare et al. 2017). Since we found an association with both diseases, FKBP5, a central element of the HPA axis, might also be an interesting target gene of such investigations. To date, there is only few evidence on a possible association of CHD or CHD risk factors with FKBP5. As CHD is robustly linked to peripheral inflammation (Kaptoge et al. 2014), and FKBP5 promotes inflammation by activating the central immune regulator NF- $\mathrm{KB}$ (Erlejman et al. 2014), it is plausible to assume that FKBP5 shapes cardiovaskular risk via this pathway. A recent study has shown that aging in combination with stress (childhood trauma or depressive phentotypes) contributes to an epigenetic up-regulation of FKBP5 (Zannas et al. 2019). These epigenetic changes were associated with increased NF- $\mathrm{\kappa B}-$ related inflammation and history of myocardial infarction. In further studies a link between FKBP5 and insulin resistance, triglyceridemia, obesity, and diabetes (Fichna et al. 2016; Gragnoli 2014; Ortiz et al. 2018) has been described. The exact role of FKBP5 in the risk for CHD and in the association of CHD with depression as well as the underlying pathophysiological mechanisms should be analyzed in further studies.

It should be taken into account that, with a mean age of 63.5 , our sample is one of elderly people. Regarding the above mentioned complex relationship between FKBP5, stress, aging, and inflammation, there is another interesting aspect: A previous review suggests that perfusion deficits in the elderly might trigger microglial activation and subsequent neuroinflammation which in turn plays a central role in the pathophysiology of late-life depression (Popa-Wagner et al. 2014). Considering the association between a HPA axis hyperactivity and the accompanying glucocorticoid resistance with inflammation (Zunszain et al. 2011), it can be assumed that FKBP5 might be associated with neuroinflammation following perfusion deficits in the elderly. However, further studies would have to investigate a possible role of FKBP5 in this context.

With regard to investigating an elderly sample, it is also important to consider that there is evidence for age specific alterations of the HPA axis itself (Deuschle et al. 1997). A systematic review investigating the HPA axis and aging in depression reports a high degree of HPA axis dysregulation in depressed older adults with differences compared with younger adults (Belvederi Murri et al. 2014). The authors proposed that this might depend on several mechanisms, including physical illnesses and alterations in the central nervous system (CNS) (Belvederi Murri et al. 2014). Regarding the CNS, it has been shown that chronic unremitting stress and the subsequent stimulation of the HPA axis in older adults often results in neuronal degeneration especially in hippocampal neuron loss (Sapolsky 1999). As a negative-feedback mediator of glucocorticoid secretion 
the hippocampus itself is essential for an effective stress response (Sapolsky 1999). Regarding the CNS in late life depression, another hypothesis has emerged in recent years: The concept of 'brain reserve' describes that some individuals might have an increased 'baseline adaptive neuroplasticity', providing greater dynamic capacity for adjusting and remodeling cortical circuits to various stressors (Freret et al. 2015). Regarding a possible role of FKBP5 in the concept of 'brain reserve' in late life depression there are two hypothesis: first, one could assume that considering the adverse effects of a dysregulated HPA axis on the brain structure, $F K B P 5$, as a central element of the HPA axis, might modulate the 'brain reserve' itself and gains its influence on depressive disorders via this pathway. Second, it is conceivable that the individual's 'brain reserve' is an independent neuroprotective factor and makes the organism more or less vulnerable to FKBP5-mediated HPA axis dysregulation and its adverse effect. Considering the 'brain reserve' as an individual and in its extent varying resilience factor, it could contribute to the inconsistent findings regarding a genetic effect of $F K B P 5$ on depressive disorders. Regarding FKBP5 and its association with pathophysiological alterations in the CNS in depressive disorders, further studies are needed.

Our finding, of carriers of the FKBP5 C allele with a more stressful CHD course displaying higher depression scores, complements the literature on the importance of $F K B P 5 \times$ environment interaction. Whereas the majority of studies investigated the $\mathrm{G} \times \mathrm{E}$ interaction with regard to the environmental factor of childhood trauma, there is only a limited number of heterogenous studies exploring stressors in adult life (Zannas et al. 2016). In the latter regard, associations of the FKBP5 minor alleles with depression and anxiety scores in patients with advanced gastric cancer (Kang et al. 2012) and with depressive symptoms in kidney transplant recipients (Shinozaki et al. 2011) were reported. Furthermore, rs $1360780 \mathrm{~T}$ allele carriers who were exposed to adult stress showed greater risk for long-term negative health implications (Lessard and Holman 2014). Interestingly, a recent study oberved that rs1360780 $\mathrm{T}$ allele carriers experienced greater post traumatic growth (PTG) following exposure to the hurricane Katrina compared to subjects with the CC genotype (Dunn et al. 2014). Other studies, however, did not find an association of $F K B P 5 \times$ adult adverse life event on risk for PTSD or depression, respectively (Binder et al. 2008; Lahti et al. 2015; Lavebratt et al. 2010).

Interestingly, in the present study a higher number of the major $\mathrm{C}$ allele was found to be associated with more depressive symptoms in the sample of CHD patients, while the majority of previous studies found the minor $\mathrm{T}$ allele to confer risk of mood and anxiety disorders (Hernandez-Diaz et al. 2019; Zannas et al. 2016). However, in line with the present findings, several studies reported an association of the major alleles of FKBP5 SNPs with depression (Zobel et al. 2010), suicidal events in depressed patients (Brent et al. 2010), and more depressive symptoms in male adolescents in the context of victimization (VanZomeren-Dohm et al. 2015). The complex nature of the FKBP5-mediated stress response, especially when taking different stressors into account, is also apparent from two studies investigating $F K B P 5 \times$ childhood trauma: whereas homozygous rs1360780 $\mathrm{T}$ allele carriers who experienced a traumatic childhood event were at the greatest risk for depression or PTSD, respectively, non-traumatized TT homozygotes were at lower risk compared to $\mathrm{C}$ allele carriers (Xie et al. 2010; Zimmermann et al. 2011). In this context, it has previously been suggested that the term 'risk gene' should be abandoned in favor of 'plasticity gene' rendering a person more vs. less sensitive to the environment (Belsky and Hartman 2014). The hypothesis of potentially beneficial effects of a hyper-responsive HPA axis is supported by another, above mentioned, study describing greater PTG in T allele carriers compared to subjects with the CC genotype after they experienced the hurricane Katrina (Dunn et al. 2014). These data are in accordance with our results as we could show that, specifically in patients with a prior MI or coronary revascularization, the $\mathrm{T}$ allele was associated with less depressive symptoms in our CHD sample.

Inconsistencies across the different studies regarding the direction of allelic association may furthermore be due to differences of the investigated stressors regarding time of exposure, duration, severity, and type (psychological vs. primarily somatic, acute vs. chronic).

In this regard, we could show that baseline ACS x FKBP5 interaction did not significantly predict depressive symptoms at the different time points. Our findings that a prior more stressful CHD course, in contrast, interacts with FKBP5 to predict depressive symptoms after hospitalization due to CHD, might be explainable by a required latency between the exposure to the CHD-related stressor and patient's depressiveness due to delayed pathophysiological alterations. Moreover, it is conceivable that only after exposure to the initial CHD-related stressor (and the assumed subsequent HPA axis dysregulation), repeated, even lower stress like the hospitalization might interact with FKBP5 on the risk for depressiveness. Accordingly, significant variability in HPA response patterns has been reported among patients with depression possibly due to different stressor characteristics such as type of stressor and duration (Burke et al. 2005; Dickerson and Kemeny 2004). The focus of previous studies investigating the FKBP5 $\times$ environment interaction was mainly the distinction into adult and childhood trauma, and specificities of the latter (Zannas et al. 2016). Our findings suggest that the exact characteristics of stressors occuring in adulthood might be central for the FKBP5 $\times$ stressor interaction on risk for depression or possibly also other stressrelated psychiatric disorders. 
Moreover, our results indicate a long-term effect of FKBP5 on patients' depressive symptoms, as FKBP5 was not only associated with patients' depressive symptoms directly after hospitalization but also after 12 months. The FKBP5 $\times$ CHD course interaction predicted depressive symptoms in the first 6 months after hospitalization, whereas it was only trendwise associated with depressive symptoms after 12 months. Those results indicate that the prior CHD course might be especially relevant for the relation of FKBP5 with depressive symptoms in the period following the hospitalization due to CHD.

A number of limitations need to be considered when interpreting the present results: The sample size is small and particularly only a few patients were homozygous for the rarer T allele. Moreover, generalizability is limited to subsyndromal depression symptoms. Referring to CHD and depressive disorders as both phenotypic heterogeneous and chronic diseases, further limitations have to be mentioned: First, although we tried to specify patients' CHD, it is still a simplistic approach regarding such a heterogenous disease, in particular, as we could show the importance of the CHD course in our present analyses. A control group without CHD would have been informative regarding the relevance of CHD for the association of FKBP5 and depressive symptoms. Second, as another central limitation, it has to be noted that episodes of depressive disorders in the patients' history were not considered. Our study showed a link between CHD and depressive symptoms depending on FKBP5 genotype, but nonetheless, information on the temporal course of both chronic diseases might be helpful to further investigate potential underlying mechanisms and lead to a more differentiated view.

Though we did not query the patient's ethnicity, the majority of patients indicated to have parents who were both born in Germany (78\%) or countries where the majority of inhabitants are also European Caucasians at the time of birth (17\%). Finally, we were not able to control for some additional factors that have been shown to be modulate the influence of $F K B P 5$ gene variation on psychiatric disorders e.g. trauma, especially in childhood, or comorbidity with other psychiatric disorders (Zannas and Binder 2014).

To our knowledge, this is the first study investigating the association of FKBP5 gene variation with depressive symptoms in the context of CHD. Overall, our study points to a relevance of $F K B P 5$ genotype in conferring depressive symptoms in CHD patients, particularly in those patients with a prior MI or coronary revascularization. Moreover, the study indicates that FKBP5 might confer a shared genetic risk for both CHD and depression. Further research will have to unravel the nature of $F K B P 5 \times$ adult stress interactions and to investigate whether determining FKBP5 genotype might aid in identifying CHD patients at risk for categorical clinical depression. This could allow for a transdiagnostic personalized medicine approach considering both cardiac and psychiatric aspects in an attempt to alleviate the individual disease burden by offering early preventive or therapeutic option for patients at risk.

Acknowledgements We thank Victoria Engelmann for her assistance in data acquisition.

Author contributions Julia Brandt and Laura Grosse drafted the manuscript and performed the statistical analyses and interpretation of the data. All authors commented on previous versions of the manuscript. Laura Grosse, Volker Arolt, Katja Beer, Wilhelm Haverkamp, Stella L. Kuhlmann, Jacqueline Müller-Nordhorn, Nina Rieckmann, Andreas Ströhle, Mira Tschorn, and Johannes Waltenberger contributed to the conception and design of the study. Volker Arolt, Katharina Warnke, Silke Jörgens, Katharina Domschke, Stella L. Kuhlmann, and Nina Rieckmann played an important role in interpreting the results. Julia Brandt, Katharina Warnke, Stella L. Kuhlmann, Kathrin Schwarte and Mira Tschorn contributed to the acquisition of data. All authors contributed to and have approved the final manuscript for publication.

Funding The current study was an add-on study to the study "Depression Care for Hospitalized Coronary Heart Disease Patients" (CDCare). CDCare was supported by the German Federal Ministry of Education and Research (Grant number: 01GY1154). The sponsor was not involved in study design, in collection, analyses and interpretation of data, in writing the article, and in the decision to submit the article for publication. Open Access funding provided by Projekt DEAL.

Availability of data The authors confirm that the data supporting the findings of this study are available within the article.

Code availability Statistical analyses were performed using IBM SPSS version 24 for Windows (SPSS Inc., Chicago, Illinois).

\section{Compliance with ethical standards}

Conflict of interest Volker Arolt declares that within the last three years of beginning this work he has received compensations for his contributions as member of advisory boards and for presentations for the following companies: Astra-Zeneca, Janssen-Organon, Lundbeck, Otsuka, Sanofi, Servier, and Trommsdorff. Wilhelm Haverkamp has been a member of the advisory boards or has given presentations on behalf of the following companies: Astra-Zeneca, Bayer, Boehringer Ingelheim, Daiichi Sankyo, Eli Lilly, Grünenthal, GlaxoSmithKline, Lundbeck, Medicines Company, MSD, Novartis, Pfizer, Trommsdorff and Servier. Andreas Ströhle received research funding from the German Federal Ministry of Education and Research, the German Research Foundation (DFG), the European Commission (FP6) and Lundbeck and speaker honoraria from AstraZeneca, Boehringer Ingelheim, Bristol-Myers Squibb, Eli Lilly \& Co, Lundbeck, Pfizer, Wyeth and UCB. He was a consultant for Actelion. Educational grants were given by the Stifterverband für die Deutsche Wissenschaft, the Berlin Brandenburgische Akademie der Wissenschaften, the Boehringer Ingelheim Fonds, the Eli Lilly International Foundation, Janssen-Cilag, Pfizer and Eli Lilly \& Co. These co-operations have no relevance to the work that is covered in the manuscript. All other authors declare that they have no conflicts of interest.

Ethics approval The study was approved by the respective ethics committees in Münster and Berlin. 
Informed consent All subjects provided written informed consent to participate in the study.

Open Access This article is licensed under a Creative Commons Attribution 4.0 International License, which permits use, sharing, adaptation, distribution and reproduction in any medium or format, as long as you give appropriate credit to the original author(s) and the source, provide a link to the Creative Commons licence, and indicate if changes were made. The images or other third party material in this article are included in the article's Creative Commons licence, unless indicated otherwise in a credit line to the material. If material is not included in the article's Creative Commons licence and your intended use is not permitted by statutory regulation or exceeds the permitted use, you will need to obtain permission directly from the copyright holder. To view a copy of this licence, visit http://creativecommons.org/licenses/by/4.0/.

\section{References}

Amare AT, Schubert KO, Klingler-Hoffmann M, Cohen-Woods S, Baune BT (2017) The genetic overlap between mood disorders and cardiometabolic diseases: a systematic review of genome wide and candidate gene studies. Transl Psychiatr. https://doi. org/10.1038/tp.2016.261

Belsky J, Hartman S (2014) Gene-environment interaction in evolutionary perspective: differential susceptibility to environmental influences. World Psychiatr. https://doi.org/10.1002/wps.20092

Belvederi Murri M, Pariante C, Mondelli V, Masotti M, Atti AR, Mellacqua Z, Antonioli M, Ghio L, Menchetti M, Zanetidou S, Innamorati M, Amore M (2014) HPA axis and aging in depression: systematic review and meta-analysis. Psychoneuroendocrinology. https://doi.org/10.1016/j.psyneuen.2013.12.004

Binder EB, Bradley RG, Liu W, Epstein MP, Deveau TC, Mercer KB, Tang Y, Gillespie CF, Heim CM, Nemeroff CB, Schwartz AC, Cubells JF, Ressler KJ (2008) Association of FKBP5 polymorphisms and childhood abuse with risk of posttraumatic stress disorder symptoms in adults. JAMA. https://doi.org/10.1001/ jama.299.11.1291

Brent D, Melhem N, Ferrell R, Emslie G, Wagner KD, Ryan N, Vitiello B, Birmaher B, Mayes T, Zelazny J, Onorato M, Devlin B, Clarke G, DeBar L, Keller M (2010) Association of FKBP5 polymorphisms with suicidal events in the Treatment of Resistant Depression in Adolescents (TORDIA) study. Am J Psychiatr. https://doi. org/10.1176/appi.ajp.2009.09040576

Burke HM, Davis MC, Otte C, Mohr DC (2005) Depression and cortisol responses to psychological stress: a meta-analysis. Psychoneuroendocrinology. https://doi.org/10.1016/j.psyneuen.2005.02.010

Caspi A, Hariri AR, Holmes A, Uher R, Moffitt TE (2010) Genetic sensitivity to the environment: the case of the serotonin transporter gene and its implications for studying complex diseases and traits. Am J Psychiatr. https://doi.org/10.1176/appi.ajp.2010.09101452

Charlson ME, Pompei P, Ales KL, MacKenzie CR (1987) A new method of classifying prognostic comorbidity in longitudinal studies: development and validation. J Clin Epidemiol 40(5):373-383. https://doi.org/10.1016/0021-9681(87)90171-8

Cohen S, Janicki-Deverts D, Miller GE (2007) Psychological stress and disease. JAMA. https://doi.org/10.1001/jama.298.14.1685

Denny WB, Valentine DL, Reynolds PD, Smith DF, Scammell JG (2000) Squirrel monkey immunophilin FKBP51 is a potent inhibitor of glucocorticoid receptor binding. Endocrinology. https://doi. org/10.1210/endo.141.11.7785

Deuschle M, Gotthardt U, Schweiger U (1997) With aging in humans the activity of the hypothalamus-pituitary-adrenal system increases and its diurnal amplitude flattens. Life Sci 61(22):2239 2246. https://doi.org/10.1016/s0024-3205(97)00926-0

Dickerson SS, Kemeny ME (2004) Acute stressors and cortisol responses: a theoretical integration and synthesis of laboratory research. Psychol Bull. https://doi. org/10.1037/0033-2909.130.3.355

Dunn EC, Solovieff N, Lowe SR, Gallagher PJ, Chaponis J, Rosand J, Koenen KC, Waters MC, Rhodes JE, Smoller JW (2014) Interaction between genetic variants and exposure to Hurricane Katrina on post-traumatic stress and post-traumatic growth: a prospective analysis of low income adults. J Affect Disord. https://doi. org/10.1016/j.jad.2013.09.018

Erlejman AG, De Leo SA, Mazaira GI, Molinari AM, Camisay MF, Fontana V, Cox MB, Piwien-Pilipuk G, Galigniana MD (2014) NF-kappaB transcriptional activity is modulated by FK506binding proteins FKBP51 and FKBP52: a role for peptidylprolyl isomerase activity. J Biol Chem. https://doi.org/10.1074/ jbc.M114.582882

Fichna M, Krzysko-Pieczka I, Zurawek M, Skowronska B, Januszkiewicz-Lewandowska D, Fichna P (2016) FKBP5 polymorphism is associated with insulin resistance in children and adolescents with obesity. Obes Res Clin Pract. https://doi.org/10.1016/j. orcp.2016.11.007

Freret T, Gaudreau P, Schumann-Bard P, Billard J, Popa-Wagner A (2015) Mechanisms underlying the neuroprotective effect of brain reserve against late life depression. J Neural Transm (Vienna) 122(1):S55-S61. https://doi.org/10.1007/s0070 2-013-1154-2

Gragnoli C (2014) Hypothesis of the neuroendocrine cortisol pathway gene role in the comorbidity of depression, type 2 diabetes, and metabolic syndrome. Appl Clin Genet. https://doi.org/10.2147/ TACG.S39993

Greenland P, Knoll MD, Stamler J, Neaton JD, Dyer AR, Garside DB, Wilson PW (2003) Major risk factors as antecedents of fatal and nonfatal coronary heart disease events. JAMA. https://doi. org/10.1001/jama.290.7.891

Hernandez-Diaz Y, Gonzalez-Castro TB, Tovilla-Zarate CA, JuarezRojop IE, Lopez-Narvaez ML, Perez-Hernandez N, RodriguezPerez JM, Genis-Mendoza AD (2019) Association between FKBP5 polymorphisms and depressive disorders or suicidal behavior: a systematic review and meta-analysis study. Psychiatr Res. https://doi.org/10.1016/j.psychres.2018.12.066

Holsboer F (2000) The corticosteroid receptor hypothesis of depression. Neuropsychopharmacology. doi: S0893-133X(00)00159-7 [pii]

Ising M, Depping AM, Siebertz A, Lucae S, Unschuld PG, Kloiber S, Horstmann S, Uhr M, Muller-Myhsok B, Holsboer F (2008) Polymorphisms in the FKBP5 gene region modulate recovery from psychosocial stress in healthy controls. Eur J Neurosci. https:// doi.org/10.1111/j.1460-9568.2008.06332.x

Jokinen J, Nordstrom P (2009) HPA axis hyperactivity and cardiovascular mortality in mood disorder inpatients. J Affect Disord. https ://doi.org/10.1016/j.jad.2008.10.025

Kang JI, Chung HC, Jeung HC, Kim SJ, An SK, Namkoong K (2012) FKBP5 polymorphisms as vulnerability to anxiety and depression in patients with advanced gastric cancer: a controlled and prospective study. Psychoneuroendocrinology. https://doi.org/10.1016/j. psyneuen.2012.02.017

Kaptoge S, Seshasai SR, Gao P, Freitag DF, Butterworth AS, Borglykke A, Di Angelantonio E, Gudnason V, Rumley A, Lowe GD, Jorgensen T, Danesh J (2014) Inflammatory cytokines and risk of coronary heart disease: new prospective study and updated metaanalysis. Eur Heart J. https://doi.org/10.1093/eurheartj/eht367

Kendler KS, Karkowski LM, Prescott CA (1999) Causal relationship between stressful life events and the onset of major depression. Am J Psychiatr. https://doi.org/10.1176/ajp.156.6.837 
Kuhlmann SL, Tschorn M, Arolt V, Beer K, Brandt J, Grosse L, Haverkamp W, Muller-Nordhorn J, Rieckmann N, Waltenberger J, Warnke K, Hellweg R, Strohle A (2017) Serum brain-derived neurotrophic factor and stability of depressive symptoms in coronary heart disease patients: a prospective study. Psychoneuroendocrinology. https://doi.org/10.1016/j.psyneuen.2016.12.015

Lahti J, Ala-Mikkula H, Kajantie E, Haljas K, Eriksson JG, Raikkonen K (2015) Associations between self-reported and objectively recorded early life stress, FKBP5 polymorphisms, and depressive symptoms in midlife. Biol Psychiatr. https://doi.org/10.1016/j. biopsych.2015.10.022

Lavebratt C, Aberg E, Sjoholm LK, Forsell Y (2010) Variations in FKBP5 and BDNF genes are suggestively associated with depression in a Swedish population-based cohort. J Affect Disord. https ://doi.org/10.1016/j.jad.2010.02.113

Lessard J, Holman EA (2014) FKBP5 and CRHR1 polymorphisms moderate the stress-physical health association in a national sample. Health Psychol. https://doi.org/10.1037/a0033968

Major Depressive Disorder Working Group of the Psychiatric GWAS Consortium, Ripke S, Wray NR, Lewis CM, Hamilton SP, Weissman MM, Breen G, Byrne EM (2013) A mega-analysis of genome-wide association studies for major depressive disorder. Mol Psychiatr. https://doi.org/10.1038/mp.2012.21

McCaffery JM, Duan QL, Frasure-Smith N, Barhdadi A, Lesperance F, Theroux P, Rouleau GA, Dube MP (2009) Genetic predictors of depressive symptoms in cardiac patients. Am J Med Genet B Neuropsychiatr Genet. https://doi.org/10.1002/ajmg.b.30824

GBD 2013 DALYs and HALE Collaborators, Murray CJ, Barber RM, Foreman KJ, Abbasoglu OA, Abd-Allah F, Abera SF, Aboyans V (2015) Global, regional, and national disability-adjusted life years (DALYs) for 306 diseases and injuries and healthy life expectancy (HALE) for 188 countries, 1990-2013: quantifying the epidemiological transition. Lancet. https://doi.org/10.1016/ S0140-6736(15)61340-X

Nicolaides NC, Charmandari E, Chrousos GP, Kino T (2014) Recent advances in the molecular mechanisms determining tissue sensitivity to glucocorticoids: novel mutations, circadian rhythm and ligand-induced repression of the human glucocorticoid receptor. BMC Endocr Disord. https://doi.org/10.1186/1472-6823-14-71

Ortiz R, Joseph JJ, Lee R, Wand GS, Golden SH (2018) Type 2 diabetes and cardiometabolic risk may be associated with increase in DNA methylation of FKBP5. Clin Epigenetics. https://doi. org/10.1186/s13148-018-0513-0

Pariante CM, Lightman SL (2008) The HPA axis in major depression: classical theories and new developments. Trends Neurosci. https ://doi.org/10.1016/j.tins.2008.06.006

Popa-Wagner A, Buga AM, Tica AA, Albu CV (2014) Perfusion deficits, inflammation and aging precipitate depressive behaviour. Biogerontology. https://doi.org/10.1007/s10522-014-9516-1

Rao S, Yao Y, Ryan J, Li T, Wang D, Zheng C, Xu Y, Xu Q (2016) Common variants in FKBP5 gene and major depressive disorder (MDD) susceptibility: a comprehensive meta-analysis. Sci Rep. https://doi.org/10.1038/srep32687

Rudisch B, Nemeroff CB (2003) Epidemiology of comorbid coronary artery disease and depression. Biol Psychiatr. https://doi. org/10.1016/S0006-3223(03)00587-0

Sapolsky R (1999) Glucocorticoids, stress, and their adverse neurological effects: relevance to aging. Exp Gerontol 34(6):721-732

Shinozaki G, Jowsey S, Amer H, Biernacka J, Colby C, Walker D, Black J, Rundell J, Stegall M, Mrazek DA (2011) Relationship between FKBP5 polymorphisms and depression symptoms among kidney transplant recipients. Depress Anxiety. https://doi. org/10.1002/da.20879

Sullivan PF, Daly MJ, O'Donovan M (2012) Genetic architectures of psychiatric disorders: the emerging picture and its implications. Nat Rev Genet. https://doi.org/10.1038/nrg3240
Touma C, Gassen NC, Herrmann L, Cheung-Flynn J, Bull DR, Ionescu IA, Heinzmann JM, Knapman A, Siebertz A, Depping AM, Hartmann J, Hausch F, Schmidt MV, Holsboer F, Ising M, Cox MB, Schmidt U, Rein T (2011) FK506 binding protein 5 shapes stress responsiveness: modulation of neuroendocrine reactivity and coping behavior. Biol Psychiatr. https://doi.org/10.1016/j.biops ych.2011.07.023

VanZomeren-Dohm AA, Pitula CE, Koss KJ, Thomas K, Gunnar MR (2015) FKBP5 moderation of depressive symptoms in peer victimized, post-institutionalized children. Psychoneuroendocrinology. https://doi.org/10.1016/j.psyneuen.2014.10.003

Wang Q, Shelton RC, Dwivedi Y (2018) Interaction between early-life stress and FKBP5 gene variants in major depressive disorder and post-traumatic stress disorder: a systematic review and meta-analysis. J Affect Disord. https://doi.org/10.1016/j.jad.2017.08.066

Whiteford HA, Degenhardt L, Rehm J, Baxter AJ, Ferrari AJ, Erskine HE, Charlson FJ, Norman RE, Flaxman AD, Johns N, Burstein R, Murray CJ, Vos T (2013) Global burden of disease attributable to mental and substance use disorders: findings from the Global Burden of Disease Study 2010. Lancet. https://doi.org/10.1016/ S0140-6736(13)61611-6

Whooley MA, Wong JM (2013) Depression and cardiovascular disorders. Annu Rev Clin Psychol. https://doi.org/10.1146/annurevclinpsy-050212-185526

Xie P, Kranzler HR, Poling J, Stein MB, Anton RF, Farrer LA, Gelernter J (2010) Interaction of FKBP5 with childhood adversity on risk for post-traumatic stress disorder. Neuropsychopharmacology. https://doi.org/10.1038/npp.2010.37

Zannas AS, Binder EB (2014) Gene-environment interactions at the FKBP5 locus: sensitive periods, mechanisms and pleiotropism. Genes Brain Behav. https://doi.org/10.1111/gbb.12104

Zannas AS, Wiechmann T, Gassen NC, Binder EB (2016) Genestress-epigenetic regulation of FKBP5: clinical and translational implications. Neuropsychopharmacology. https://doi.org/10.1038/ npp. 2015.235

Zannas AS, Jia M, Hafner K, Baumert J, Wiechmann T, Pape JC, Arloth J, Kodel M, Martinelli S, Roitman M, Roh S, Haehle A, Emeny RT, Iurato S, Carrillo-Roa T, Lahti J, Raikkonen K, Eriksson JG, Drake AJ, Waldenberger M, Wahl S, Kunze S, Lucae S, Bradley B, Gieger C, Hausch F, Smith AK, Ressler KJ, MullerMyhsok B, Ladwig KH, Rein T, Gassen NC, Binder EB (2019) Epigenetic upregulation of FKBP5 by aging and stress contributes to NF-kappaB-driven inflammation and cardiovascular risk. Proc Natl Acad Sci USA. https://doi.org/10.1073/pnas.1816847116

Zigmond AS, Snaith RP (1983) The hospital anxiety and depression scale. Acta Psychiatr Scand 67(6):361-370. https://doi. org/10.1111/j.1600-0447.1983.tb09716.x

Zimmermann P, Bruckl T, Nocon A, Pfister H, Binder EB, Uhr M, Lieb R, Moffitt TE, Caspi A, Holsboer F, Ising M (2011) Interaction of FKBP5 gene variants and adverse life events in predicting depression onset: results from a 10-year prospective community study. Am J Psychiatr. https://doi.org/10.1176/appi.ajp.2011.10111577

Zobel A, Schuhmacher A, Jessen F, Hofels S, von Widdern O, Metten M, Pfeiffer U, Hanses C, Becker T, Rietschel M, Scheef L, Block W, Schild HH, Maier W, Schwab SG (2010) DNA sequence variants of the FKBP5 gene are associated with unipolar depression. Int J Neuropsychopharmacol. https://doi.org/10.1017/S1461145709991155

Zunszain PA, Anacker C, Cattaneo A, Carvalho LA, Pariante CM (2011) Glucocorticoids, cytokines and brain abnormalities in depression. Prog Neuropsychopharmacol Biol Psychiatr. https:// doi.org/10.1016/j.pnpbp.2010.04.011

Publisher's Note Springer Nature remains neutral with regard to jurisdictional claims in published maps and institutional affiliations. 